\title{
Modelling the Power Consumption in Femtocell Networks
}

\author{
Margot Deruyck, Dieter De Vulder, Wout Joseph, and Luc Martens \\ Ghent University/IBBT, Dept. of Information Technology \\ Gaston Crommenlaan 8 box 201, B-9050 Ghent, Belgium \\ Email: margot.deruyck@intec.ugent.be
}

\begin{abstract}
In this paper, the energy efficiency of a femtocell base station is investigated and compared for various bit rates and for three different wireless technologies namely, mobile WiMAX, HSPA, and LTE. A power consumption model is proposed and applied in a network deployment tool to develop energyefficient femtocell networks. Furthermore, it is investigated to what extent the introduction of sleep modes can reduce the power consumption in femtocell networks. Reductions of $\mathbf{2 4 \%}$ are obtained when sleep modes are introduced into the network.
\end{abstract}

\section{INTRODUCTION}

Today, a lot of people buy or already possess one or more mobile and thus wireless devices. The Wireless World Research Forum (WWRF) has a vision of 7 trillion wireless devices serving 7 billion users by 2017 [1]. These mobile devices are currently not only used outdoor, but are also used in indoor environments. Therefore, the operators and the manufacturers start searching for solutions to increase indoor coverage. One possibility is the introduction of femtocell base stations. A femtocell base station is rather cheap and can be installed by the end user himself/herself. It communicates with the cellular network through a broadband connection such as DSL (Digital Subscriber Line) or cable modem, or through a separate RF (Radio Frequency) backhaul channel, thereby reducing the traffic on the cellular network.

The power consumption of one femtocell base station is limited, however, when hundreds or thousands femtocell base stations are used in a network, the power consumption can become significant. Furthermore, Informa Telecoms \& Media concludes in its study on the occasion of the World Mobile Congress in Barcelona 2011 that there will be 49 million femtocell access points serving 114 million users in 2014 [2]. It is thus clear that it is interesting to investigate the power consumption of these femtocell base stations.

The purpose of this paper is to investigate the energy efficiency of femtocell base stations and compare it for various bit rates and three different wireless technologies namely, mobile WiMAX (Worldwide Interoperability for Microwave Access), HSPA (High Speed Packet Access), and LTE (Long Term Evolultion). Therefore, a power consumption model is proposed. Based on this model, a deployment tool is designed which develops energy-efficient femtocell networks (i.e., with a minimal power consumption) for a predefined area. In a last step, it is investigated to what extent the power consumption can be reduced by introducing sleep modes in the developed networks.

The outline of the paper is as follows: in Section II the power consumption model is described. Section III gives more information about the deployment tool we developed. In Section IV, results obtained with the power consumption model and the deployment tool are discussed and sleep modes are introduced into the network. Finally, Section V summarizes the most important conclusions.

\section{ENERGY EFFICIENCY OF A FEMTOCELL}

To allow the comparison of the performance of the different technologies considered, the energy efficiency is used. Here, the energy efficiency is defined as the power consumption needed to cover a certain area (in $\mathrm{W} / \mathrm{m}^{2}$ ) [3], [4], [5]:

$$
P C_{\text {area }}=\frac{P_{e l}}{\pi \cdot R^{2}}
$$

with $P_{e l}$ the power consumption of the femtocell (in W) and $R$ the range of the femtocell (in $\mathrm{m}$ ). The lower $P C_{\text {area }}$, the more energy-efficient the femtocell is. How $P_{e l}$ and $R$ are determined, is described in Section II-A and II-B, respectively.

\section{A. Power consumption model}

The development of the power consumption model is based on the hardware model for a femtocell base station of [6]. Three interacting blocks can be defined in a femtocell base station. The first block consists of the microprocessor which is responsible for the implementation and the management of the standardised radio protocols and the associated base band processing. Furthermore, it is also responsible of managing the backhaul connection to the core network. The second block is the FPGA (Field-Programmable Gate Array), along with some other integrated circuitry to support a variety of functions such as data-encryption, hardware authentication and the Network Time Protocol. The third block contains the RF (Radio Frequency) transmitter which allows to send and receive signals and the power amplifier.

Based on this hardware model, a function is derived for the power consumption $P_{e l}$ of the femtocell base station (in W):

$$
P_{e l / f e m t o}=P_{e l / m p}+P_{e l / F P G A}+P_{e l / t r a n s}+P_{e l / a m p}
$$

with $P_{e l / m p}, P_{e l / F P G A}, P_{e l / t r a n s}$ and $P_{e l / a m p}$ the power consumption (in $\mathrm{W}$ ) of, respectively, the microprocessor, the FPGA, the transmitter and the power amplifier. 
The power consumption of each block is constant throughout time and thus a fixed number is used for the power consumption [6]. To determine the power consumption of the power amplifier $P_{e l / a m p}$ (in $\mathrm{W}$ ) the following equation is used [3], [4]:

$$
P_{e l / a m p}=\frac{P_{T x}}{\eta}
$$

wit $P_{T x}$ the input power of the antenna (in $\mathrm{W}$ ) and $\eta$ the efficiency of the power amplifier which is the ratio of the RF output power to the electrical input power. $\eta$ can take values between $5 \%$ and $40 \%$. Here, a power amplifier is assumed with a medium performance (i.e., 22.5\%) for $\eta$. The values for the other parameters can be found in Table I.

\begin{tabular}{|l|c|c|}
\hline Component & & Value \\
\hline Microprocessor & $P_{e l / m p}$ & $3.2 \mathrm{~W}$ \\
\hline FPGA & $P_{e l / F P G A}$ & $4.7 \mathrm{~W}$ \\
\hline Transmitter & $P_{e l / t r a n s}$ & $1.7 \mathrm{~W}$ \\
\hline Efficiency & $\eta$ & $22.5 \%$ \\
\hline
\end{tabular}

TABLE I

POWER CONSUMPTION OF THE FEMTOCELL BASE STATION COMPONENTS [6].

\section{B. Determination of the range}

To determine the range of a femtocell base station, a link budget needs to be composed [3], [4]. A link budget takes all of the gains and losses of the transmitter through the medium to the receiver into account. Firstly, the maximum allowable path loss $P L_{\max }$ a transmitted signal can experience while still being detectable at the receiver is determined. Table II gives an overview of the link budget parameters for the considered technologies.

\begin{tabular}{|l|c|c|c|}
\hline Parameter & Mobile WiMAX & HSPA & LTE \\
\hline Frequency [MHz] & 2500 & 2100 & 2600 \\
\hline $\begin{array}{l}\text { Maximum input power antenna } \\
\text { of base station [dBm] }\end{array}$ & 23 & 15 & 21 \\
\hline Antenna gain of base station [dBi] & 2 & 2 & 2 \\
\hline Antenna gain of mobile station [dBi] & 2 & 0 & 0 \\
\hline Soft handover gain of mobile station [dB] & 0 & 1.5 & 0 \\
\hline Feeder loss of base station [dB] & 0.5 & 0 & 2 \\
\hline Feeder loss of mobile station [dB] & 0 & 0 & 0 \\
\hline Fade margin [dB] & 6 & 7.7 & 4 \\
\hline Interference margin [dB] & 2 & 2 & 2 \\
\hline Bandwidth [MHz] & 5 & 5 & 5 \\
\hline Receiver SNR [dB] & {$[6,8.5,11.5$,} & {$[-3.1,0.1,3.4$,} & {$[-1.5,3,10.5$} \\
& $151921]^{1}$ & $6,7.1,9.6$ & $14,19,23$ \\
& & $15.6]^{2}$ & $23,29.4]^{3}$ \\
\hline Number of used subcarriers & 360 & - & 301 \\
\hline Number of total subcarriers & 512 & - & 512 \\
\hline Noise figure of mobile station [dB] & 7 & 7 & 5 \\
\hline Implementation loss of mobile station [dB] & 2 & 0 & 0 \\
\hline Shadowing margin [dB] & 13.2 & 13.2 & 13.2 \\
\hline Duplexing & TDD & TDD & TDD \\
\hline
\end{tabular}

(1) format: [1/2 QPSK, 3/4 QPSK, 1/2 16-QAM, 3/4 16-QAM, 2/3 64-QAM, 3/4 64-QAM]

2) format: [1/4 QPS, 1/2 QPSK, 3/4 QPSK, 3/4 8-QAM, 1/2 16-QAM, 3/4 16-QAM, 3/4 64-QAM]

(3) format: [1/3 QPSK, 1/2 QPSK, 2/3 QPSK, 1/2 16-QAM, 2/3 16-QAM, 4/5 16-QAM, 1/2 64-QAM, 2/3 64-QAM]

TABLE II

LINK BUDGET TABLE FOR THE TECHNOLOGIES CONSIDERED.

Once $P L_{\max }$ is known, the range can be calculated by using a propagation model. As femtocell base stations are situated indoor, the most appropriate propagation model is the ITUR P.1238 model [7]. This model predicts the path loss for three possible scenarios: a residential building, a commercial building or an office. Here, the office scenario is assumed. The range $R$ is then determined as follows:

$$
R=10 \frac{P L_{\max }-20 \cdot \log _{10} f-L_{f}(n)+28}{N}
$$

with $f$ the frequency (in $\mathrm{MHz}$ ), $L_{f}(n)$ the floor penetration loss factor $(\mathrm{dB}), n$ the number of floors between base station and terminal and $N$ the distance power loss coefficient. As the office scenario is here considered, $L_{f}(n)$ corresponds with $15+4 \cdot(n-1)$ and $N$ equals 30 .

\section{DEPLOYMENT TOOL}

The model described in Section II is used in a deployment tool. This deployment tool allows to design energy-efficient femtocell networks (i.e., with a minimal power consumption) to provide indoor coverage in a predefined area, here noted as the target area, and is based on the GRAND (Green Radio Access Network Design) as defined in [8]. This area is supplied as a shape file [8]. This shape file gives more information about the buildings in the area and their position, form and height.

Firstly, the tool determines a set of possible locations for the femtocell base stations. Each building is a possible location of a femtocell base station. This base station is placed at the centre of the building. In this way, the size of the set is somewhat limited to reduce the simulation duration. Initially, no femtocell base stations are active.

Secondly, the tool computes an energy-efficient network based on these locations. Therefore, a genetic search algorithm is used. The algorithm will generate a first set of possible solutions, the so-called population, by activating femtocell base stations. The size of the population is specified by the end user. Based on this first population, the algorithm will optimize the solutions through a number of generations. A new generation of solution is created by mutating (some of) the solutions of the previous generation, by selecting the best solutions of the previous solution and by the crossover operator. Four mutations are defined: (i) activating an inactive femtocell base station, (ii) deactivating an active femtocell base station, (iii) adding $1 \mathrm{dBm}$ to the input power of an antenna of an active femtocell base station, and (iv) subtracting $1 \mathrm{dBm}$ of the input power of an antenna of an active femtocell base station. Which femtocell base station and how it is adapted, is randomly determined. To determine if a new solution is better than the previous one, two different fitness functions are defined. One tells how good a solution performs in terms of coverage of the target area and the second in terms of power consumption. The coverage fitness returns the percentage of the indoor target area that is covered by the solution. The maximum power consumption of the network (i.e., when each possible location contains a femtocell base station) is used as reference. The model of Section II is used to determine the power consumption fitness. These two fitness functions are combined into one global fitness function by using a weighted average of the coverage fitness and the power consumption fitness. The same functions as in [3], [8] are used. Furthermore, these functions are also used to select the best solutions 
in a generation. The crossover operator allows to create a new hopefully better solution by using different parts of two mediocre or bad solutions.

The algorithm will continue to create new solutions until a stopping criterion is met. This stopping criterion is set by the end user. The algorithm will stop if a certain amount of generations is created or if the maximum allowable simulation duration is exceeded. The longer the simulation duration, the better the solution will be.

\section{Results}

\section{A. Comparison of the energy efficiency}

In this section, the energy efficiency of the considered technologies, namely, mobile WiMAX, HSPA, and LTE is compared for various bit rates in a $5 \mathrm{MHz}$ channel at the frequencies specified in Table II. The office scenario is assumed with $n$ equals to 1 (i.e., only one floor between base station and receiver). Fig. 1 compares $P C_{\text {area }}$ of the technologies considered for different bit rates in a $5 \mathrm{MHz}$ channel.

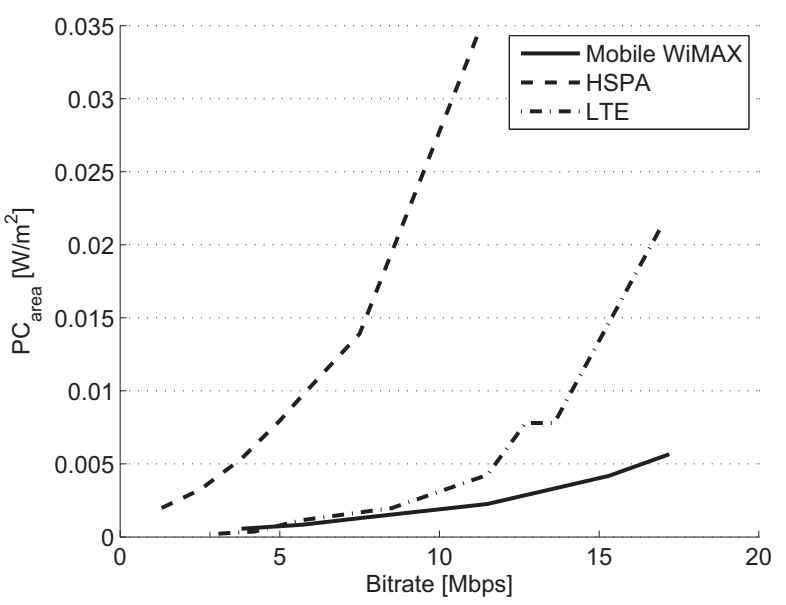

Fig. 1. Comparison of $P C_{a r e a}$ for the technologies considered in a $5 \mathrm{MHz}$ channel.

The femtocell base station consumes $10.5 \mathrm{~W}, 10.0 \mathrm{~W}$, and 9.7 W, respectively, for mobile WiMAX, LTE, and HSPA. The differences in power consumption are due to the differences in input power of the antenna (Table II). The higher the input power of the antenna, the higher the power consumption of the power amplifier (eq. (3)) and thus the higher the power consumption of the base station.

For bit rates higher than $5 \mathrm{Mbps}$, mobile WiMAX is the most energy-efficient technology $\left(P C_{\text {area }}=34.9 \mathrm{~mW} / \mathrm{m}^{2}\right.$ versus $4.2 \mathrm{~mW} / \mathrm{m}^{2}$ for LTE and $2.3 \mathrm{~mW} / \mathrm{m}^{2}$ for HSPA and for a bit rate of approximately $11 \mathrm{Mbps})$. Although mobile WiMAX has a higher power consumption, it obtains a higher range $(38.5 \mathrm{~m}$ versus $27.4 \mathrm{~m}$ and $9.4 \mathrm{~m}$ for LTE and HSPA respectively) due to its lower receiver SNR (Signal-to-Noise Ratio) and its higher input power of the antenna (Table II), resulting thus in a higher energy efficiency.

For bit rates between $2.8 \mathrm{Mbps}$ and $5 \mathrm{Mbps}$, LTE is the most energy-efficient technology $\left(P C_{\text {area }}=0.4 \mathrm{~mW} / \mathrm{m}^{2}\right.$ versus
$0.6 \mathrm{~mW} / \mathrm{m}^{2}$ and $5.4 \mathrm{~mW} / \mathrm{m}^{2}$ for, respectively, mobile WiMAX and HSPA for a bit rate of approximately $4 \mathrm{Mbps}$ ). Again, a higher range is obtained with LTE $(93.5 \mathrm{~m}$ versus $76.8 \mathrm{~m}$ and $24 \mathrm{~m}$ for mobile WiMAX and HSPA respectively and for a bit rate of approximately $4 \mathrm{Mbps}$ ) then for the other technologies due to its lower receiver SNR for the considered bit rates (Table II).

Finally, HSPA is the most energy-efficient technology for bit rates below the $2.8 \mathrm{Mbps}$ as these bit rates are not supported by the other technologies.

\section{B. Application: a energy-efficient femtocell network for a part of Ghent}

In this section, it is investigated how much power is needed to cover a certain part of Ghent by femtocells. The deployment tool described in Section III is used for this purpose. Fig. 2 gives an overview of the target area and the possible locations for femtocell base stations in this area. The surface of this area is about $0.11 \mathrm{~km}^{2}$ from which $60.78 \%$ is covered by buildings and where coverage needs to be foreseen. In this area, 495 possible locations for the femtocell base stations are found (i.e., in each building a femtocell base station can be placed).

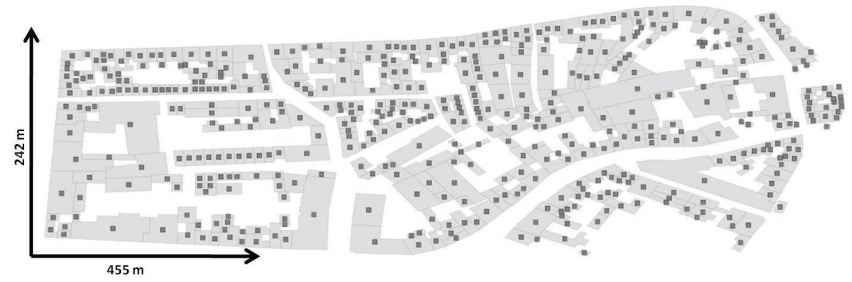

Fig. 2. Overview of the target area and the possible locations (squares) for femtocell base stations in the target area $\left(0.11 \mathrm{~km}^{2}\right)$.

Fig. 3 gives an overview of the solution network for mobile WiMAX (Fig. 3(a)), HSPA (Fig. 3(b)), and LTE (Fig. 3(c)). A bit rate of $5 \mathrm{Mbps}$ is considered in a $5 \mathrm{MHz}$ channel, to make a fair comparison between the different technologies. Furthermore, for the genetic search algorithm, the population size is chosen as 50 , a maximum of 1000 generations can be created, and the simulation duration is limited to $1 \mathrm{~h}$. However, when the estimated value of the generation is not higher than $1 \%$ compared to the previous generation, the algorithm is also stopped. The numerical results are listed in Table III for a coverage requirement of $90 \%$.

In agreement with the results from Section IV-A, mobile WiMAX performs the best. For a coverage of approximately $98 \%$, the WiMAX network only consumes $0.4 \mathrm{~kW}$, resulting in the lowest value for $P C_{\text {area }}$.

\section{Introduction of sleep modes}

The power consumption of the femtocell networks designed in Section IV-B can be further minimized by introducing sleep modes. When there is little or no activity in its cell, the 


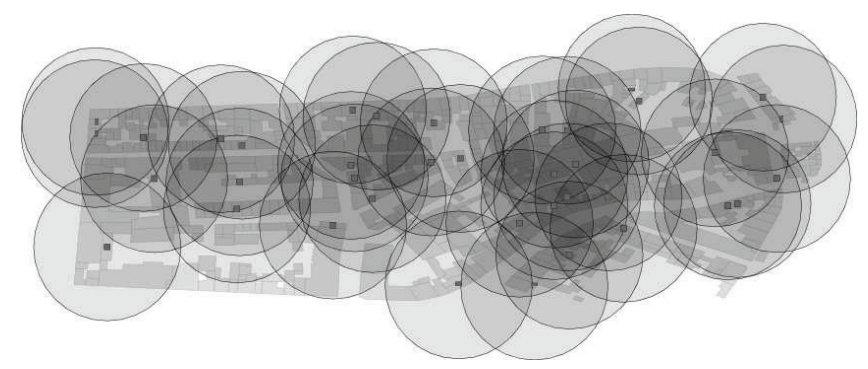

(a)

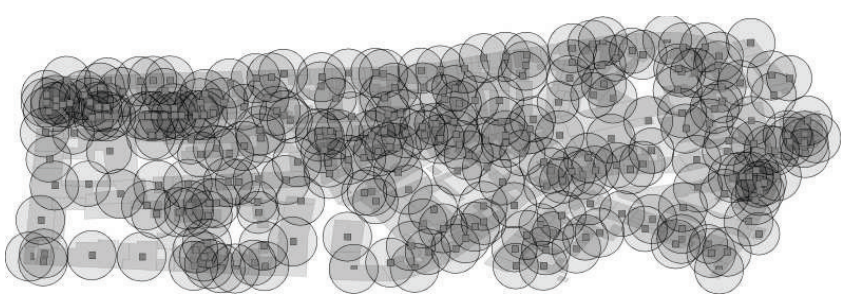

(b)

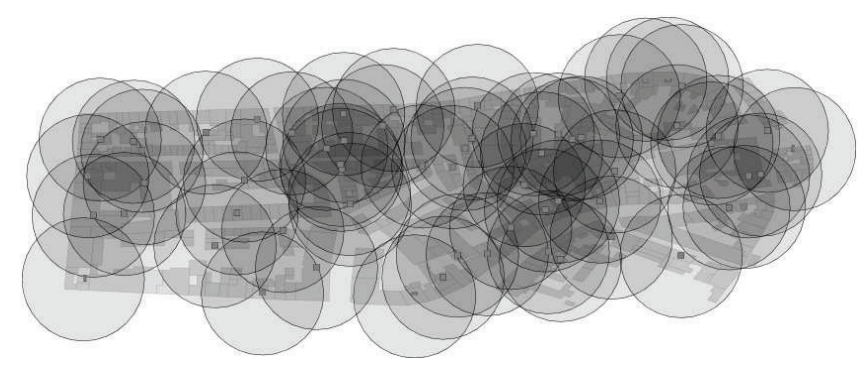

(c)

Fig. 3. Energy-efficient femtocell networks for mobile WiMAX (a), HSPA (b), and LTE (c) for a bit rate of $5 \mathrm{Mbps}$ in a $5 \mathrm{MHz}$ channel resulting from the deployment tool.

\begin{tabular}{|l|c|c|c|}
\hline Parameter & $\begin{array}{c}\text { Mobile } \\
\text { WiMAX }\end{array}$ & HSPA & LTE \\
\hline Number of femtocells used & 38 & 272 & 63 \\
\hline Power consumption [kW] & 0.4 & 2.6 & 0.6 \\
\hline Coverage percentage [\%] & 97.6 & 95.9 & 99.5 \\
\hline$P C_{\text {area }}\left[\mathrm{mW} / \mathrm{km}^{2}\right]$ & 3.7 & 25.0 & 5.8 \\
\hline
\end{tabular}

TABLE III

COMPARISON OF THE ENERGY-EFFICIENT FEMTOCELL NETWORK FOR THE TECHNOLOGIES CONSIDERED AND A PHYSICAL BIT RATE OF 5 MBPS.

femtocell base station can enter the sleep mode i.e., a mode wherein the femtocell base station consumes only a small amount of power. When it is necessary, the femtocell base station can be awaken to serve the users in its cell. In [6], three sleep mode techniques are proposed: the sleep mode is controlled by either the femtocell base station itself, or by the core network, or by the UE (user equipment). In this paper, the UE controlled approach will be used as this technique does not need the overlaying macrocell network for signalling the wake-up signals. In the UE controlled approach, the UE can broadcast wake-up signals when it is necessary to wake up a femtocell base station. Although the femtocell base station is in sleep mode, it retains the capability to receive wake-up signals from the UE and, if necessary, to switch to an active state. In the sleep mode, the RF transmitter $(1.0 \mathrm{~W}[6])$, the power amplifier $(0.9 \mathrm{~W}, 0.03 \mathrm{~W}$, and $0.4 \mathrm{~W}$ for, respectively, mobile WiMAX, HSPA, and LTE) and some other circuit elements (1.7 W [6]) can be switched off, leading to a reduction in power consumption of $3.6 \mathrm{~W}, 2.8 \mathrm{~W}$, and $3.1 \mathrm{~W}$ for, respectively, mobile WiMAX, HSPA, and LTE.

\section{1) Performance of the sleep mode technique:}

In this section, it is investigated how much power is saved by introducing the sleep mode for one femtocell base station. In the next section, it is studied what the power consumption reduction is in the networks developed in Section IV-B.

The same procedure as in [9] is used for this investigation. The average power consumption reduction of the femtocell base station (as a percentage) is defined as [9]:

$$
\Omega=\frac{P_{\text {sleep }}}{P_{\text {active }}} \cdot(1-\bar{\eta}) \cdot 100
$$

with $P_{\text {sleep }}$ the power consumption of the femtocell base station in sleep mode (in W), $P_{\text {active }}$ the power consumption of the femtocell base station in active mode (in W) and $\bar{\eta}(0 \leq \bar{\eta} \leq 1)$ the average duty cycle (i.e., the time that the femtocell base station spends in the active mode). To determine $\bar{\eta}$ a traffic model is used [9]. This traffic model indicates when there are periods of high and low traffic during the day. Typically, it is modelled by a Poisson process with exponentially distributed arrival time and exponentially distributed service time (mean value is here chosen to be $3 \mathrm{~min}$ ). The traffic model used is based on the traffic model proposed in [9] but it is adapted to the office environment here considered (Section II). Table IV gives an overview of the traffic model used in this study.

\begin{tabular}{|l|c|c|c|}
\hline Time & Usage & $\begin{array}{c}\text { Arrival time } \lambda \\
{[\text { min.] }}\end{array}$ & $\begin{array}{c}\text { Traffic/user } \\
{[\text { Erlang] }}\end{array}$ \\
\hline $0 \mathrm{~h}-8 \mathrm{~h}$ & Low & 240 & 0.0125 \\
\hline $8 \mathrm{~h}-18 \mathrm{~h}$ & High & 30 & 0.1 \\
\hline $18 \mathrm{~h}-0 \mathrm{~h}$ & Low & 240 & 0.0125 \\
\hline
\end{tabular}

TABLE IV

TRAFFIC MODEL FOR AN OFFICE ENVIRONMENT.

Fig. 4 illustrates the results for $\Omega$ for the technologies considered. The maximum power consumption reduction is between $29 \%$ and $34 \%$ when no users are present. This is a theoretical case as it is not realistic that the femtocell base station sleeps all day. The highest reduction is obtained with mobile WiMAX as the power consumption of the power amplifier is the highest for this base station (as it has the highest input power of the antenna, Table II) and thus more power can be saved in sleep mode compared to HSPA and LTE. Furthermore, the more users are present in the cell, the lower the power consumption reduction is. This is logical due to the fact that when there are more users in a cell, the femtocell base station can sleep less, resulting in a lower reduction. A femtocell base station has typically between 1 to 16 users. Based on the results from Fig. 4, it is concluded that 
the sleep mode introduction is very useful until a boundary of 8 users. For more than 8 users, there is still a power consumption reduction, however, this reduction becomes very small $(2-3 \%)$.

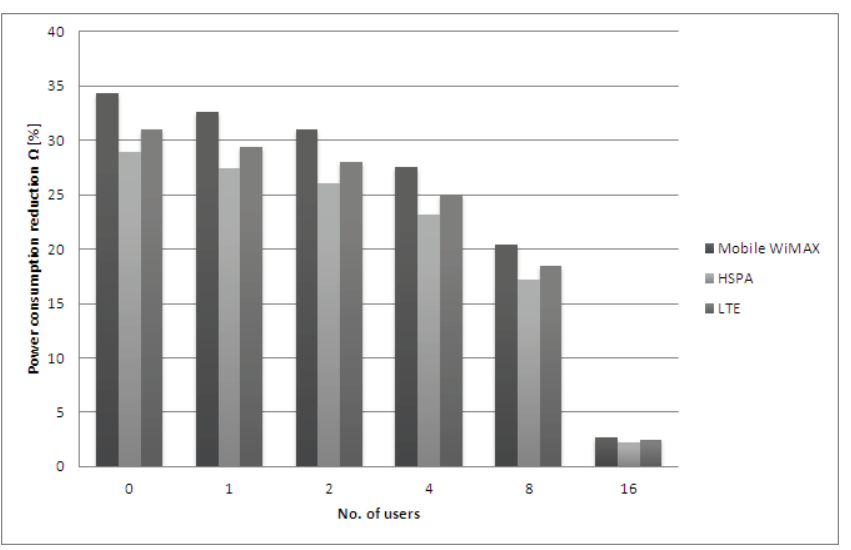

Fig. 4. Power consumption reduction for the femtocell base station of the technologies considered and for various numbers of users supported.

2) Power consumption reduction in femtocell networks:

Now, it is determined how much power can be saved in the networks developed in Section IV-B. Therefore, a random number of users between 1 and 8 is allocated to each cell in the network. Based on this number and the results from Section IV-C1, it is calculated how the dialy power consumption is reduced for each femtocell in the network and thus what the overall power consumption reduction for the whole network is. Table $\mathrm{V}$ gives an overview of the results from this study.

\begin{tabular}{|l|c|c|c|}
\hline Parameter & $\begin{array}{c}\text { Mobile } \\
\text { WiMAX }\end{array}$ & HSPA & LTE \\
\hline No. femtocells & 38 & 273 & 64 \\
\hline No. users in network & 198 & 1180 & 280 \\
\hline $\begin{array}{l}\text { Average no. user } \\
\text { per femtocell }\end{array}$ & 5.2 & 4.3 & 4.4 \\
\hline $\begin{array}{l}\text { Dialy power consumption } \\
\text { without sleep modes [kWh] }\end{array}$ & 9.6 & 63.3 & 15.12 \\
\hline $\begin{array}{l}\text { Daily power consumption } \\
\text { with sleep modes [kWh] }\end{array}$ & 7.1 & 49.0 & 11.5 \\
\hline Power consumption reduction & $25.4 \%$ & $22.7 \%$ & $24.2 \%$ \\
\hline
\end{tabular}

TABLE V

POWER CONSUMPTION REDUCTION IN FEMTOCELL NETWORKS FOR THE TECHNOLOGIES CONSIDERED.

Introducing sleep modes in the network results in a power consumption reduction between $22 \%$ and $25 \%$. The highest reduction is achieved with mobile WiMAX which corresponds with the results obtained in Section IV-C1. The number of users served is the highest for HSPA as HSPA has the highest number of femtocell base stations in its network. However, the average number of users per femtocell is approximately the same (i.e., 4.6 users per femtocell) for the three technologies considered. If the same number of users is assumed for each network, the network with the highest number of femtocell base stations will correspond with the highest power consumption reduction as it will be possible to let more femtocell base stations sleep. Therefore, the average number of users per femtocell is kept (approximately) the same in this investigation.

\section{CONCLUSION}

In this paper, the energy efficiency of femtocell base stations is investigated and compared for three wireless technologies namely, mobile WiMAX, HSPA, and LTE. Therefore, a power consumption model is proposed. Based on this model, it was found that a femtocell base station consumes, in general, approximately $10 \mathrm{~W}$ for a range between 9 to $130 \mathrm{~m}$ (depending on the technology and the bit rate considered). For the parameters assumed and a $5 \mathrm{MHz}$ channel, mobile WiMAX is the most energy-efficient technology for bit rates higher than 5 Mbps. For bit rates between $2.8 \mathrm{Mbps}$ and $5 \mathrm{Mbps}$, LTE is the most energy-efficient.

The power consumption model is then used in a deployment tool for the development of energy-efficient femtocell networks. For the area considered and a bit rate of $5 \mathrm{Mbps}$, the mobile WiMAX consumes $105.6 \mathrm{kWh}$ per day, the HSPA network $499.8 \mathrm{kWh}$, and the LTE network $146.4 \mathrm{kWh}$.

Furthermore, sleep modes are introduced in the network. The sleep mode technique reduces the power consumption significantly when it supports a maximum of 8 users. For a higher number of users, there is still a power consumption reduction but this reduction is rather small $(<3 \%$ for the power consumption of a single femtocell base station). Introducing sleep modes in the network leads to a power consumption reduction of approximately $24 \%$.

\section{ACKNOWLEDGMENT}

The work described in this paper was carried out with the support of the IBBT-project GreenICT. W. Joseph is a Post-Doctoral Fellow of the FWO-V (Research Foundation Flanders).

\section{REFERENCES}

[1] World Wireless Research Forum, WWRF, www.wireless-worldresearch.org.

[2] D. Duffy, Femtocell market set for strong growth in 2011, Informa Telecoms \& Media, 2010

[3] M. Deruyck, E. Tanghe, W. Joseph, L. Martens, Modelling and optimization of power consumption in wireless access networks, Elsevier Computer Communications, 2011, doi: 10.1016/j.comcom.2011.03.008.

[4] M. Deruyck, E. Tanghe, W. Joseph, W. Vereecken, M. Pickavet, L. Martens, B. Dhoedt, Model for Power Consumption of Wireless Access Networks, IET Science, Measurement \& Technology, Vol. 5, Issue 4, July 2011, pp. 155-161.

[5] L. M. Correia, D. Zeller, O. Blume, D. Ferling, Y. Jading, I. Gódor, G. Auer, L. Van der Perre, Challenges and Enabling Technologies for Energy Aware Mobile Radio Networks, IEEE Communications Magazine, Vol. 48, Issue 11, November 2010, pp. 66-72.

[6] I. Ashraf, F. Boccardi, L. Ho, Power Savings in Small Cell Deployments via Sleep Mode Techniques, 21st Annual IEEE Symposium on Personal, Indoor and Mobile Radio Communications (PIMRC 2010): Workshop W-Green, Istanbul, Turkey, September 2010, pp. 306-310.

[7] Recommendation ITU-R P.1238-2, Propagation data and prediction methods for the planning of indoor radiocommunication systems and radio local area networks in the frequency range $900 \mathrm{MHz}$ to $100 \mathrm{GHz}$, 1997-1999-2001. 
[8] M. Deruyck, E. Tanghe, W. Joseph, W. Vereecken, M. Pickavet,

B. Dhoedt, L. Martens, Towards a deployment tool for wireless access networks with minimal power consumption, 21st Annual IEEE Symposium on Personal, Indoor and Mobile Radio Communications (PIMRC 2010): Workshop W-Green, Istanbul, Turkey, September 2010, pp. 294-299.

[9] I. Ashraf, L. Ho, H. Claussen, Improving Energy Efficiency of Femtocell Base Stations via User Activity Dection, Wireless Communications and Networking Conference (WCNC 2010), Sydney, Australia, April 2010, pp. $1-5$. 